\title{
Basic Association Rules
}

\author{
Guichong Li and Howard J. Hamilton \\ Department of Computer Science \\ University of Regina \\ Regina, SK, Canada, S4S 0A2 \\ \{liguicho, hamilton\}@cs.uregina.ca
}

\begin{abstract}
Previous approaches for mining association rules generate large sets of association rules. Such sets are difficult for users to understand and manage. Here, the concept of a restricted conditional probability distribution is used to explain an association rule. Based on this concept, a new type of association rules, called basic association rules, is defined. We propose the GenBR algorithm to generate the set of classes of basic association rules. Theoretical analysis shows that the search space of the algorithm can be translated to an $n$ cube graph. The set of classes of basic association rules generated by GenBR is easy for users to understand and manage. Our experiments on synthetic and real datasets show that GenBR is either faster than previous approaches or generates fewer rules or both.
\end{abstract}

Keywords: Association Rules, Probabilistic/Statistical Methods, Data Reduction/Preprocessing, Restricted Conditional Probability Distributions, Inference Systems, and Basic Association Rules.

\section{Introduction}

Techniques for mining association rules [1,2] were originally devised for application to market basket data, but they have also been applied in many other domains to perform tasks $[21,23,26]$. Market basket data describes the items purchased from retail stores grouped into transactions. A transaction typically consists of items bought together at the same point of time, but it may consist of items bought by a customer over a period of time. An itemset is a set of items, and a frequent itemset $X$ is an itemset whose frequency in transactions, also referred to as its support, denoted as $\operatorname{supp}(X)$, is greater than a user specified support threshold, minsup.

The main task of association rule discovery is to extract frequent itemsets from market basket data and to generate association rules from these frequent itemsets. An association rule $r$ is an implication of the form $X \rightarrow Y$, where $X$ and $Y$ are two disjoint itemsets. The support of the rule is the support of $X \cup Y$, denoted as $\operatorname{supp}(r)$, which is given by the observed probability $P(X=1, Y=1)$. The confidence of the rule, denoted $\operatorname{conf}(r)$, is given by the conditional observed probability $P(X=1, Y=1) / P(X=$ $1)$, which is denoted as $p(x y) / p(x)$ in this paper. If an association rule has support at least as great as minsup and confidence at least as great as the confidence threshold called minconf, it is referred to as a valid association rule. An association rule with confidence $100 \%$ is an exact association rule; all other association rules are approximate association rules.

The Apriori algorithm [2] was proposed to discover all frequent itemsets and to generate all valid association rules corresponding to these itemsets by a fast algorithm, called FastGenRules. Many algorithms have since been proposed that reduce the time and space required to find the frequent itemsets $[2,14]$. After all frequent itemsets have been found, valid association rules are generated.

A serious problem in association rule discovery is that the set of association rules can grow to be unwieldy as the number of transactions increases, especially if the support and confidence thresholds are small. As the number of frequent itemsets increases, the number of rules presented to the user typically increases proportionately. Many of these rules may be redundant. The definition of "redundancy" for association rules has varied in previous approaches. Toivonen et al. proposed finding a structural rule cover, which describes the same database rows as the original set of association rules [28]. Therefore, those rules that are not in the cover are regarded as redundant. In $[11,20,24,30]$, the definition of redundant rules is based on several inference rules or an inference system. Therefore, all association rules that can be derived from other rules by applying inference rules are regarded as redundant. We adopt the latter type of definition.

To address the problem of rule redundancy, four types of research on mining association rules have been performed. First, rules have been extracted based on userdefined templates or item constraints [3,27]. Secondly, researchers have developed interestingness measures to select only interesting rules [16,18,19]. Thirdly, researchers have proposed inference rules or inference systems to prune redundant rules and thus present smaller, and usually more understandable sets of association rules to the user $[5,11,20,24,30]$. Finally, new frameworks for mining association rule have been proposed that find association rules with different formats or properties $[7,8,9]$.

The main problems with previous approaches are that they still generate too many rules, and these rules may be 
redundant. For example, a valid association rule $X \rightarrow Y$ that is generated by one these approaches may in fact be derived from some simpler rule $X^{\prime} \rightarrow Y^{\prime}$ with the same confidence as $X \rightarrow Y$, where $X^{\prime} \subseteq X$ and $Y^{\prime} \subseteq Y$. Inference rules proposed by these approaches do not resemble Armstrong axioms on functional dependencies. As well, in some approaches, inference rules cannot infer the confidence of rules without extra information.

In our research, we are creating an inference system on association rules, consisting of a set of inference rules such as augmentation and transitivity, which resembles the Armstrong axioms on functional dependencies and which allows the inference of the confidences of rules.

The remainder of this paper is organized as follows. In Section 2, we present related work. In Section 3, we define the concept of a basic association rule, and propose a new algorithm called GenBR for generating the set $B R$ of classes of basic association rules from a set of frequent itemsets. The computational complexity of GenBR is also described. A comparison of our approach and other approaches is presented in Section 4. Our experiments compared the performance of our algorithm with that of previous algorithms on synthetic datasets and real-life datasets, with respect to the number of rules and the elapsed running time. Conclusions and future work are described in Section 5.

\section{Previous Work}

Previous research showed that relatively small sets of association rules can be presented to users instead of all valid association rules. As well, for some approaches, inference rules were suggested that allowed additional association rules to be derived from such small sets of rules. In this section, we describe three approaches.

First, representative association rules $(R R)$ are based on a cover operator with which other non-representative association rules can be generated [20]. Suppose we have an association rule $X \rightarrow Y$. A cover operator $C$, denoted $C(X \rightarrow Y)$, is given by

$$
\begin{aligned}
C(X \rightarrow Y)= & \{X \cup Z \rightarrow V \mid Z, V \subseteq Y \wedge Z \cap V=\varnothing \wedge \\
V \neq \varnothing\} &
\end{aligned}
$$

The set of all representative association rules is a minimal set of rules that covers all association rules by means of the cover operator. The FastGenRepresentative algorithm was proposed to efficiently compute a $R R$ [20].

Secondly, a kind of non-redundant association rules with minimal antecedents and maximal consequents, called minimal non-redundant association rules, has been identified as particularly useful and relevant [5]. An association rule $r: X \rightarrow Y$ is a minimal non-redundant association rule iff there does not exist an association rule $r^{\prime}: X^{\prime} \rightarrow Y^{\prime}$ with $\operatorname{supp}(r)=\operatorname{supp}\left(r^{\prime}\right), \operatorname{conf}(r)=\operatorname{conf}\left(r^{\prime}\right), X^{\prime} \subseteq$ $X$ and $Y \subseteq Y^{\prime}$. A small non-redundant generating set for all valid association rules is formed by combining a generic basis $G B$ for exact association rules and an informative basis $I B$ for approximate association rules. $R I$ is defined as a transitive reduction of the informative basis corresponding to $I B$. Given a closure operator $c$ of the Galois connection, a set $F C$ of frequent closed itemsets, the set $G$ of their generators, and a partial order $\prec$ (inclusion relation) on the set of itemsets, the definitions of $G B, I B$ and $R I$ are as follows.

$$
\begin{aligned}
& G B=\left\{r: g \rightarrow(f \backslash g) \mid f \in F C \wedge g \in G_{f} \wedge g \neq f\right\} \\
& I B=\{r: g \rightarrow(f \backslash g) \mid f \in F C \wedge g \in G \wedge c(g) \subset f\} \\
& R I=\{r: g \rightarrow(f \backslash g) \mid f \in F C \wedge g \in G \wedge c(g) \prec f \wedge \\
& \left.\quad \nexists f^{\prime} c(g) \prec f^{\prime} \prec f\right\}
\end{aligned}
$$

Bastide et al. have proven that $G B$ and $I B$ contain only minimal non-redundant association rules and all exact association rules and approximate association rules can be derived from $G B$ and $I B$, respectively [5]. The Gen-GB and Gen-RI algorithms were proposed to generate a generic basis and a transitive reduction of the informative basis, respectively. According to the definition of a minimal non-redundant association rule, the support and confidence of any association rules inferred from the generating set are the same as the support and confidence of the rules from which they were inferred. The authors claim that none of the Armstrong axioms hold in non-redundant association rules. A similar approach has been proposed for discovering a small cover for association rules based on closed itemsets, which adapts the Duquenne-Guigues basis for exact association rules and the Luxenburger results for approximate association rules [24].

Thirdly, informative cover has been proposed together with a new inference rule [11]. Let $r, r^{\prime}$ be two association rules, denoted $X \rightarrow Y$ and $X^{\prime} \rightarrow Y^{\prime}$, such that $X^{\prime}$ $\cup Y^{\prime} \subseteq X \cup Y$. If $\operatorname{supp}\left(X^{\prime}\right) \leq \operatorname{supp}(X)$, we say that $r$ covers $r^{\prime}$, denoted $r \prec r^{\prime}$. The goal is to find an informative cover that covers all other association rules. The CoverRules algorithm has been proposed to generate an informative cover for association rules [11].

The cover operator in the informative cover approach is similar to the cover operator in the representative association rule approach. The difference between them is that the cover operator of the informative cover approach does not require the antecedent of the resulting association rule to be included in the antecedent of the initial association rule. In addition, the inference procedure is not purely syntactic [11], because it uses

Table 2.1. A Binary Dataset.

\begin{tabular}{lllll}
\hline$A$ & $B$ & $C$ & $D$ & $E$ \\
\hline 1 & 0 & 1 & 1 & 1 \\
0 & 0 & 1 & 1 & 1 \\
1 & 0 & 1 & 1 & 1 \\
1 & 1 & 1 & 1 & 0 \\
0 & 1 & 0 & 1 & 1 \\
1 & 1 & 0 & 1 & 0 \\
\hline
\end{tabular}


information about the support of the antecedent of the resulting association rule. The inference rules in the two approaches are sound, but neither approach infers the confidence of an association rule.

We found that the rules generated by these approaches may be redundant. If $X \rightarrow Y$ is generated by any of these approaches, it may be possible to derive it from simpler valid association rules.

Example 2.1. Suppose we have the dataset shown in Table 2.1, and that minsup is 0.3 and minconf is 0.6. The sets of rules generated by the previous approaches are shown in Table 2.2.

Consider the rules in Table 2.2 from the perspective of a user. For $R R, C E \rightarrow A D$ can be derived from simpler association rules $C E \rightarrow A$ and $C E \rightarrow D$ by right union as with Armstrong axioms, and $\operatorname{conf}(C E \stackrel{0.67}{\rightarrow} A D)=$ $0.67 \quad 1.0$ $\operatorname{conf}(C E \rightarrow A) \times \operatorname{conf}(C E \rightarrow D)$. For GB, $A B \rightarrow D$ is unnecessary, because $B \rightarrow D$ is in $G B$. For $C, B \rightarrow A D$ can be derived from $B \rightarrow A$ and $B \rightarrow D$ by right union, assuming $B \rightarrow A$ and $B \rightarrow D$ are valid association rules. Transitivity cannot be used between $G B$ and $R I$. For example, $E \rightarrow D$ in $G B$ and $D \rightarrow A$ in $R I$ cannot be used to infer a valid association rule by transitivity. These examples show that some association rules in these generating sets are not in the most desirable form.

\section{Discovery of Basic Association Rules}

We propose a new approach to solve the problems mentioned in Section 2.

Table 2.2. The Generated Association Rules

\begin{tabular}{|c|c|c|c|}
\hline Algorithm & Set & Rule & \#Rule \\
\hline FastGenRules & $A R$ & $\ldots$ & 36 \\
\hline FastGenRR & $R R$ & 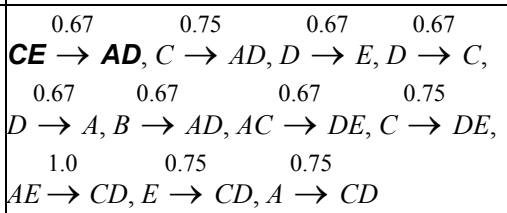 & 11 \\
\hline \multirow[t]{2}{*}{$\begin{array}{l}\text { Gen-GB } \\
\text { and Gen-RI }\end{array}$} & $G B$ & 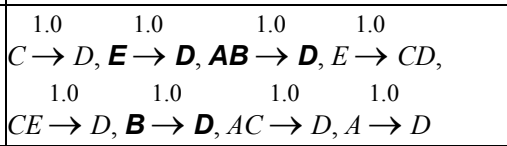 & 8 \\
\hline & $R I$ & 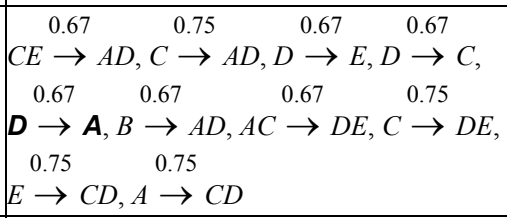 & 10 \\
\hline CoverRules & $C$ & $\begin{array}{l}\stackrel{0.75}{E \rightarrow} C D, D \stackrel{0.67}{\rightarrow} E, C \stackrel{0.75}{\rightarrow} A D, D \rightarrow C \\
0.67\end{array}$ & 7 \\
\hline
\end{tabular}

\subsection{Definitions}

Definition 3.1.1. Given a dataset $D$ with $I$ as a set of items and $T$ as a set of transactions, an association rule $\stackrel{p}{\rightarrow} Y$ over a relation $\mathrm{R} \subseteq I \times T$ is said to be in canonical form if $|Y|=1$.

According to this definition, we only consider the case of $Y$ containing a single item. Before we introduce other new notions, let us discuss another concept related to conditional probability.

A conditional probability distribution (CPD) $P(Y \mid X)$ is defined as $P(X, Y) / P(X)$, where $X$ and $Y$ are random variables [10]. $Y$ is conditionally independent of $Z$ given $X$, denoted as $I(Y, Z \mid X)$, if and only if $P(Y \mid X)=P(Y \mid X$, $Z$ ), where $X, Y$, and $Z$ are three disjoint sets of random variables. The statement $I(X, Z \mid X)$ is referred to as a conditional independence statement (CIS) [10].

Four properties that are satisfied by any joint probability distribution (JPD) are symmetry, decomposition, weak union, and contraction [10]. For example, for the decomposition property, if $I(X, Y \cup W \mid$ $Z)$, then $I(X, Y \mid Z)$ and $I(X, W \mid Z)$.

We describe a CIS in another way in Lemma 3.1.1.

Lemma 3.1.1. Given a subset $X^{\prime} \subset X$, we say $I\left(Y, X \backslash X^{\prime} \mid\right.$ $X^{\prime}$ ), i.e., $Y$ is conditionally independent of $X \backslash X^{\prime}$ given $X^{\prime}$, if and only if $P(Y \mid X)=P\left(Y \mid X^{\prime}\right)$, where $X$ and $Y$ are two disjoint sets of variables.

Proof:

Suppose $Z=X \backslash X^{\prime}$. Then $X^{\prime}, Y$, and $Z$ are three disjoint sets of variables. Since $X=X^{\prime} \cup Z, P(Y \mid X)=P(Y$ $\left.\mid X^{\prime}, Z\right)$. Assuming $P(Y \mid X)=P\left(Y \mid X^{\prime}\right)$, we derive $P\left(Y \mid X^{\prime}\right.$, $Z)=P\left(Y \mid X^{\prime}\right)$. Thus, we have $I\left(Y, Z \mid X^{\prime}\right)$, i.e., $I\left(Y, X \backslash X^{\prime} \mid\right.$ $\left.X^{\prime}\right)$ according to the definition of $C I S$.

For the converse, assuming $I\left(Y, X \backslash X^{\prime} \mid X^{\prime}\right)$, then according to the definition of $C I S$, we have $P\left(Y\left|X^{\prime}, X\right|\right.$ $\left.X^{\prime}\right)=P\left(Y \mid X^{\prime}\right)$, i.e., $P(Y \mid X)=P\left(Y \mid X^{\prime}\right)$.

Furthermore, we have Lemma 3.1.2.

Lemma 3.1.2. Given two disjoint sets of variables $X$ and $Y$, and $X^{\prime} \subset X$, if $I\left(Y, X \backslash X^{\prime} \mid X^{\prime}\right)$, then $\forall Z \subseteq X \backslash X^{\prime}, I(Y, Z \mid$ $\left.X^{\prime}\right)$, i.e., $\forall Z \subseteq X \backslash X^{\prime}, P(Y \mid X)=P\left(Y \mid X^{\prime}\right)=P\left(Y \mid X^{\prime}, Z\right)$.

Proof:

If $Z=X \backslash X^{\prime}$, the proof follows immediately. The decomposition property of conditional independencies states that if $I(X, Y \cup W \mid Z)$, then $I(X, Y \mid Z)$ and $I(X, W \mid$ $Z$ ). Because $I\left(Y, X \backslash X^{\prime} \mid X^{\prime}\right), \forall Z \subset X \backslash X^{\prime}$, we obtain $I(Y$, $\left.Z \mid X^{\prime}\right)$ and $I\left(Y,\left\{X \backslash X^{\prime}\right\} \backslash Z \mid X^{\prime}\right)$. From $I\left(Y, X \backslash X^{\prime} \mid X^{\prime}\right)$, we obtain $P(Y \mid X)=P\left(Y \mid X^{\prime}, X \backslash X^{\prime}\right)=P\left(Y \mid X^{\prime}\right)$, and from $I(Y$, $\left.Z \mid X^{\prime}\right)$, we obtain $P\left(Y \mid X^{\prime}\right)=P\left(Y \mid X^{\prime}, Z\right)$. Hence, $P(Y \mid X)$ $=P\left(Y \mid X^{\prime}\right)=P\left(Y \mid X^{\prime}, Z\right)$.

To explain this idea more fully, we first give a more general definition as follows.

Definition 3.1.2. Given two disjoint sets of variables $X$ and $Y$, and a conditional probability distribution $P(Y \mid X)$, a restricted conditional probability distribution $(R C P D)$, 
denoted as $\hat{P}(\hat{Y} \mid \hat{X})$, is a subset of $P(Y \mid X)$ defined by specifying subsets $\operatorname{Domain}(\hat{Y}) \subseteq \operatorname{Domain}(Y)$ and $\operatorname{Domain}(\hat{X}) \subseteq \operatorname{Domain}(X)$.

For binary variables $X \in\{0,1\}$ and $Y \in\{0,1\}$, with $\hat{X} \in\{1\}$ and $\hat{Y} \in\{1\}$, the confidence $p(y \mid x)$ of the association rule $r: X \rightarrow Y$ is a positive conditional probability of the $R C P D \hat{P}(\hat{Y} \mid \hat{X})$.

In the following discussion, $\hat{P}(\hat{Y} \mid \hat{X})$ is simply denoted as $\hat{P}(Y \mid X)$.

Given $X^{\prime} \subset X$, if we have $\hat{P}(Y \mid X)=\hat{P}\left(Y \mid X^{\prime}\right)$, we cannot guarantee that $\forall Z \subseteq X \backslash X^{\prime}, \hat{P}(Y \mid X)=\hat{P}\left(Y \mid X^{\prime}\right.$, $Z$ ). Hence, the decomposition property cannot be applied in a RCPD.

Example 3.1.1. Suppose that we have the transaction dataset in Table 2.1 and that minsup is 0.3 and minconf is 0.6. Consider two association rules $A C D \rightarrow E$ and $D \rightarrow E$. Although $\operatorname{conf}(A C D \rightarrow E)=\operatorname{conf}(D \rightarrow E)=2 / 3, \operatorname{conf}(C D E)$ $=3 / 4$, i.e., if $X=\{A, C, D\}$ and $Y=\{E\}$. If we choose $X^{\prime}$ $=\{D\}$, then $Z=X \backslash X^{\prime}=\{A, C\}, p(y \mid x)=p\left(y \mid x^{\prime}, z\right)=p(y \mid$ $\left.x^{\prime}\right)=2 / 3$, i.e., $\hat{P}(Y \mid X)=\hat{P}\left(Y \mid X^{\prime}\right)$. Let $Z=\{C\} \subset X \backslash X^{\prime}$, since $p\left(y \mid x^{\prime}, z\right)=3 / 4$, then $p(y \mid x) \neq p\left(y \mid x^{\prime}, z\right)$ and $p(y \mid$ $\left.x^{\prime}\right) \neq p\left(y \mid x^{\prime}, z\right)$, i.e., $\hat{P}(Y \mid X) \neq \hat{P}\left(Y \mid X^{\prime}, Z\right)$ and $\hat{P}\left(Y \mid X^{\prime}\right)$ $\neq \hat{P}\left(Y \mid X^{\prime}, Z\right)$.

In the context of RCPDs, we hope to find a minimal subset $X^{\prime}$ of $X$ with respect to $Y$ such that $\hat{P}(Y \mid X)=\hat{P}(Y$ $\left.\mid X^{\prime}\right)$, and $\forall Z \subseteq X \backslash X^{\prime}, \hat{P}(Y \mid X)=\hat{P}\left(Y \mid X^{\prime}, Z\right)$.

Definition 3.1.3. Let $X$ and $Y$ be two disjoint sets of variables. $X^{\prime}$ is a minimal conditional subset (MCS) of $X$ with respect to $Y$ if $X^{\prime}$ is a subset of $X$ that satisfies the following conditions:

(1) $\hat{P}(Y \mid X)=\hat{P}\left(Y \mid X^{\prime}\right)$.

(2) $\forall Z \subseteq X \backslash X^{\prime}, \hat{P}(Y \mid X)=\hat{P}(Y \mid X, Z)$.

(3) $\nexists X^{\prime \prime} \subset X^{\prime}$ such that conditions (1) and (2) hold for $X^{\prime \prime}$.

If $X$ is a minimal conditional subset of itself with respect to $Y$, then $X$ is conditionally minimal with respect to $Y$. For example, in Table $2.1, A C$ is conditionally minimal with respect to $E$.

We also define the dual.

Definition 3.1.4. Suppose $X$ and $Y$ are disjoint sets of variables. If $X^{\prime}$ is a MCS of $X$ with respect to $Y$, then the restricted conditional probability distribution $\hat{P}\left(\hat{Y} \mid \hat{X}^{\prime}\right)$ is a minimal conditional probability distribution $(M C P D)$ of the $\operatorname{RCPD} \hat{P}(\hat{Y} \mid \hat{X})$ with respect to $\hat{Y}$. If $X$ is conditionally minimal with respect to $Y$, then $\hat{P}(\hat{Y} \mid \hat{X})$ is a MCPD of itself.

If $\hat{P}\left(\hat{Y} \mid \hat{X}^{\prime}\right)$ is a MCPD of $\hat{P}(\hat{Y} \mid \hat{X})$, then a series of equations follow, i.e., $\forall Z \subseteq X \backslash X^{\prime}, \hat{P}(\hat{Y} \mid \hat{X})=$ $\hat{P}\left(\hat{Y} \mid \hat{X}^{\prime}, \hat{Z}\right)$.
Because MCS and MCPD are duals of each other, we use whichever is convenient.

In previous research, MCS and MCPD have not been defined or emphasized by researchers. In the context of mining association rules, we use a RCPD $\hat{P}(Y \mid X)$ for inference on association rules. We do not consider how $\hat{P}(X Y)$ and $\hat{P}\left(X^{\prime} Y\right)$ behave.

Example 3.1.2. Suppose we have the transaction dataset shown in Table 2.1. Let $X=\{A, C, D\}, Y=\{E\}, X^{\prime}=\{A$, $C\}$. Because the confidences of both $X \rightarrow Y$ and $X^{\prime} \rightarrow Y$ are $2 / 3$, i.e., the positive conditional probability $p(e \mid a c d)=$ $p(e \mid a c)$, we see that $\hat{P}(Y \mid X)=\hat{P}\left(Y \mid X^{\prime}\right)$ and that $\forall Z \subseteq$ $X \backslash X^{\prime}=\{D\}, \hat{P}(Y \mid X)=\hat{P}\left(Y \mid X^{\prime}, Z\right)$. Because $p(e \mid a c d)$ $\neq p(e \mid a)=2 / 4$ and $p(e \mid a c d) \neq p(e \mid c)=3 / 4$, there is no $X^{\prime \prime} \subset X^{\prime}=\{A, C\}$ such that $\hat{P}(Y \mid X)=\hat{P}\left(Y \mid X^{\prime \prime}\right)$. So $X^{\prime}$ is a MCS of $X$ with respect to $Y$. $\hat{P}\left(Y \mid X^{\prime}\right)$ is a MCPD of $\hat{P}(Y \mid X)$ with respect to $Y$.

Similarly, $\{A\},\{C\}$, and $\{E\}$ are three MCSs of $\{A$, $C, E\}$ with respect to $D$.

In the context of mining association rules, a CPD $P(Y$ $\mid X)$ is always referred to as a $\operatorname{RCPD} \hat{P}(\hat{Y} \mid \hat{X})$.

We define a new notion of minimal association rules analogously to minimal functional dependencies [25].

Definition 3.1.5. Suppose we have a set $I$ of items and a transaction dataset $D$. A canonical association rule $X \stackrel{p}{\rightarrow} Y$ over $D$ is a basic association rule if $X$ is conditionally minimal with respect to $Y$, i.e., $\nexists X^{\prime} \subset X$ such that

(1) $P(Y \mid X)=P\left(Y \mid X^{\prime}\right)$ and

(2) $\forall Z \subseteq X \backslash X^{\prime}, P(Y \mid X)=P\left(Y \mid X^{\prime}, Z\right)$.

For example, given the transaction dataset shown in Table 2.1, $A C \rightarrow E$ is a basic association rule, and $A C$ is a MCS of $A C D$ with respect to $E$ while $P(E \mid A C)$ is a MCPD of $P(E \mid A C E)$ with respect to $E$.

\subsection{Computing MCPDs}

According to the definition of basic association rules, either a MCS $X$ with respect to $Y$ or a MCPD $P(Y \mid X)$ corresponds to a basic association rule $X \rightarrow Y$. The confidence of the rule is a positive conditional probability of $P(Y \mid X)$. Therefore, the crucial task of finding basic association rules is the computation of all MCPDs.

Given a set $L$ of frequent itemsets, $\forall X \in L$, and minconf, our approach for computing MCPDs corresponding to $X$ is divided into two steps. We first construct a set of RCPDs in canonical form from $X$, and then we compute their MCPDs, in which all positive conditional probabilities are at least as great as minconf.

Our approach is similar to the approach for discovering the minimal directed I-Map of a joint probability distribution (JPD) [10]. Suppose that a permutation (ordering) $Y=\left\{Y_{1}, \ldots, Y_{n}\right\}$ of a set of variables $X=\left\{X_{1}, \ldots, X_{n}\right\}$, and $p(x)$ is a JPD of $X$. This approach computes any minimal set of predecessors $\prod_{i}$ with respect 
to $Y_{i}$, and $\prod_{i}$ satisfies $p\left(y_{i} \mid b_{i}\right)=p\left(y_{i} \mid \pi_{i}\right)$, where $\prod_{i} \subseteq B_{i}=$ $\left\{Y_{1}, \ldots, Y_{i-1}\right\}$. Hence, a directed minimal $I$-map of $p(x)$ is constructed by designating $\prod_{i}$ as parents of $Y_{i}$. The differences from computing I-map from a JPD is that we do not permutate items of a frequent itemset, and the conditions (contexts) in the restricted conditional probabilities contain all items in the frequent itemset except for a single test item.

For example, to find MCPDs corresponding to the frequent itemset $X_{1} X_{2} X_{3} X_{4} X_{5}$, first the following RCPDs are constructed:

$P\left(X_{1} \mid X_{2} X_{3} X_{4} X_{5}\right), P\left(X_{2} \mid X_{1} X_{3} X_{4} X_{5}\right), P\left(X_{3} \mid X_{1} X_{2} X_{4} X_{5}\right)$, $P\left(X_{4} \mid X_{1} X_{2} X_{3} X_{5}\right)$, and $P\left(X_{5} \mid X_{1} X_{2} X_{3} X_{4}\right)$.

For $P\left(X_{1} \mid X_{2} X_{3} X_{4} X_{5}\right)$, one can observe all MCSs of $X_{2} X_{3} X_{4} X_{5}$ with respect to $X_{1}$, such as a minimal subset $\Pi$ $\subseteq\left\{X_{2} X_{3} X_{4} X_{5}\right\}$, and $\forall Z \subseteq\left\{X_{2} X_{3} X_{4} X_{5}\right\} \backslash \prod, p\left(x_{1} \mid x_{2} x_{3} x_{4} x_{5}\right)$ $=p\left(x_{1} \mid \pi\right)=p\left(x_{1} \mid \pi, z\right)$, where $P\left(X_{1} \mid \Pi\right)$ is a MCPD of $P\left(X_{1} \mid X_{2} X_{3} X_{4} X_{5}\right)$. If $p\left(x_{1} \mid x_{2} x_{3} x_{4} x_{5}\right)$ is less than minconf, then we stop the computation of MCPDs. Otherwise, $\Pi$ is a MCS of $X_{2} X_{3} X_{4} X_{5}$ with respect to $X_{1}$. The corresponding basic association rule is $\prod^{p} \rightarrow X_{1}$, as explained in Section 3.1. Similarly, we compute the MCPDs of $P\left(X_{2} \mid\right.$ $\left.X_{1} X_{3} X_{4} X_{5}\right), P\left(X_{3} \mid X_{1} X_{2} X_{4} X_{5}\right), P\left(X_{4} \mid X_{1} X_{2} X_{3} X_{5}\right)$, and $P\left(X_{5} \mid\right.$ $\left.X_{1} X_{2} X_{3} X_{4}\right)$. Finally, a set of MCPDs is obtained. Thus, for each frequent itemset, a set of basic association rules with respect to $X$ can be found.

Because RCPDs do not obey decomposition, to compute a MCPD $P\left(Y \mid X^{\prime}\right)$ of $P(Y \mid X)$, we should examine all cases, i.e., $\forall Z \subseteq X \backslash\left\{Y \cup X^{\prime}\right\}, P\left(Y \mid X^{\prime}\right)=P(Y$ $\left.\mid X^{\prime}, Z\right)$. This process goes from top to bottom, and is depicted as a semi-lattice [12] in Figure 3.1.

The frequent itemset itself, such as $\mathrm{ABCDE}$, is placed at the first level. At the second level, we construct a set of RCPDs, such as $P(A \mid B C D E), P(B \mid A C D E), P(E \mid$ $A B C D), P(D \mid A B C E)$, and $P(E \mid A B C D)$. All RCPDs at the third level are constructed by setting their contexts as maximal subsets of the contexts of the RCPDs at the second level. At the fourth level, all RCPDs are formed by setting their contexts as the intersections of the contexts of the RCPDs at the third level. The number $k$ of itemsets being intersected at level $d$ of the semi-lattice is related to the length $l$ of the frequent itemset at the first level. We have the formula $k=d-2,4 \leq d \leq l$. For example, for frequent 4 -itemsets, the number of itemsets being intersected at level 4 is 2 . For frequent 5 -itemsets, the number of itemsets being intersected at level 5 is 3 . The depth of the semi-lattice equals the size of the corresponding frequent itemset.

For example, for the computation of the MCPDs of $P(E \mid$ $A B C D)$, we first examine $P(E \mid A B C), P(E \mid A B D), P(E \mid$ $A C D)$, and $P(E \mid B C D)$. If $P(E \mid A B C D)=P(E \mid A B C)$ and $P(E \mid A B C)$ is minimal, then $P(E \mid A B C)$ is already a MCPD of $P(E \mid A B C D)$, and we examine $P(E \mid A B D), P(E$ $\mid A C D)$, and $P(E \mid B C D)$. Similar cases arise

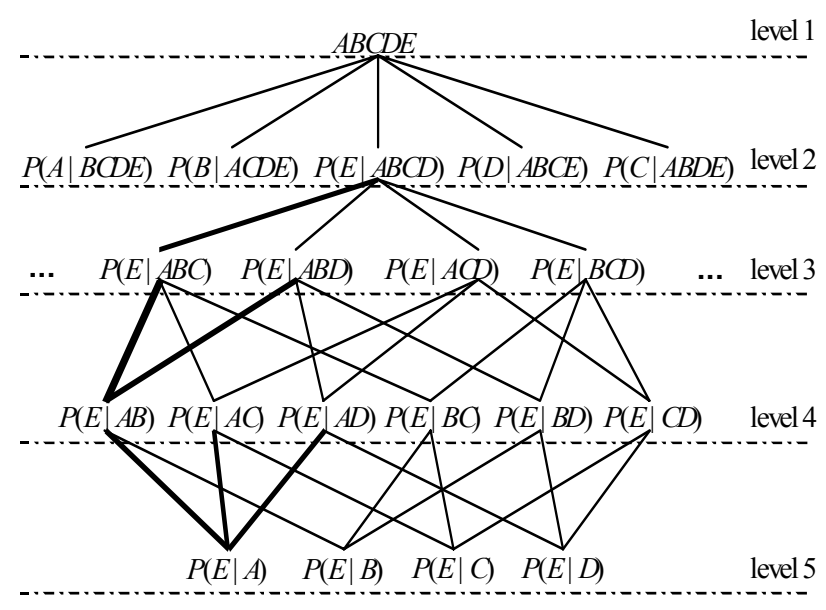

Figure 3.1. A Possible Semi-lattice for the Frequent Itemset ABCDE.

for $P(E \mid A B D), P(E \mid A C D)$, and $P(E \mid B C D)$. If $P(E \mid A B)$ is a MCPD of $P(E \mid A B C D)$, we only require $P(E \mid A B C D)$ $=P(E \mid A B C)=P(E \mid A B D)$. The context of $P(E \mid A B)$ is the intersection of the contexts of $P(E \mid A B C)$ and $P(E \mid$ $A B D)$. If $P(E \mid A)$ is a MCPD of $P(E \mid A B C D)$, we require $P(E \mid A B)=P(E \mid A C)=P(E \mid A D)=P(E \mid A B C D)$, where $P(E \mid A B), P(E \mid A C)$, and $P(E \mid A D)$ have already been obtained. Therefore, according to Definition 3.1.3 and 3.1.4, $P(E \mid A)$ is a MCPD of $P(E \mid A B C D)$.

During extension of the semi-lattice, the RCPDs in new children (the nodes at the next level) are also called the candidate MCPDs (not unique). We reduce the number of candidate MCPDs of new children by intersecting itemsets in the contexts of their parents (nodes at the previous level), and checking whether the candidate MCPDs of the children are equal to those of their parents, and whether the positive conditional probabilities of their MCPDs are at least as great as minconf.

To find all basic association rules, we should check all frequent itemsets and compute the corresponding minimal conditional probabilities.

Therefore, we define the following concepts.

Definition 3.2.1. Suppose we have a transaction dataset $D$, minsup, minconf, and a set $L$ of frequent itemsets over $D$. A class of basic association rules for $X \in L$ is a set of basic association rules $r: X^{\prime} \rightarrow A$, denoted as $C_{r}(X)$, such that

(1) $X^{\prime} \cup A \subseteq X$

(2) $X^{\prime}$ is a MCS of $X \backslash A$ with respect to $A$

(3) $\operatorname{conf}(r) \geq$ minconf

Definition 3.2.2. Suppose we have a transaction database $D$, minsup, and minconf. A basic association rule system, denoted as $B R(D$, minsup, minconf $)$, is defined as the set of $k$ distinct classes of valid basic association rules, i.e., $B R(D$, minsup, minconf $)=\left\{C_{r}^{i} \mid C_{r}^{i}\right.$ is a class of basic 
association rules, $1 \leq i \leq k$ such that for all $j, 1 \leq j \leq k, j \neq$ $\left.i, C_{r}^{i} \nsubseteq C_{r}^{j}, C_{r}^{i} \nsupseteq C_{r}^{j}\right\} . B R(D$, minsup, minconf $)$ is also simply denoted as $B R$ when $D$, minsup, and minconf are clear from context.

Example 3.2.1. Given the dataset in Table 2.1, minsup = 0.3 , and minconf $=0.6$, in Figure 3.2 , we show how to compute all MCPDs corresponding to the frequent itemset $A C D E$.

Figure 3.2 shows the search space used to find MCSs by computing a set of MCPDs corresponding to the frequent itemset $A C D E$. This search space consists of four semi-lattices, in which the single node at the top level corresponds to the frequent itemset, and other nodes correspond to its subsets, each of which includes a positive conditional probability.

Regardless of the data in the dataset, at the second level in the structure, we always have four positive conditional probabilities for $A C D E$. Each of them corresponds to an item in $A C D E$, such as $p(a \mid c d e)$, etc. At the third level of the structure, the itemsets appearing in the contexts of positive conditional probabilities are always maximal subsets of the itemsets appearing in the context of positive conditional probabilities in their parents. For example, along the branch containing $p(a)$ $c d e$ ), de, ce and $c d$ are maximal subsets of $c d e$. If the positive conditional probability of a child is equal to the positive conditional probability of its parent and both positive conditional probabilities are at least as great as minconf, then in Figure 3.2, they are connected with a bold arrow; e.g., a bold arrow is shown from the node including $p(a \mid c d e)$ to the node including $p(a \mid c e)$, because $p(a \mid c d e)=p(a \mid c e)$. If the positive conditional probability of a parent is not equal to the positive conditional probability of its child, but the positive conditional probability of the child is at least as great as minconf, we connect the parent node and the child node with a narrow arrow; e.g., a narrow arrow is shown from the node including $p(a \mid c d e)$ to the node including $p(a \mid$ $c d$ ). If the positive conditional probability of a parent is not equal to the positive conditional probability of its child or a positive conditional probability is less than minconf, further computation of minimal conditional probabilities along this path is terminated; e.g., a dotted arrow is shown from the node including $p(a \mid c d e)$ to the node including $p(a \mid d e)$. Hence, $P(A \mid C E)$ is a MCPD of $P(A \mid C D E)$.

Similarly, we compute all MCPDs of $P(C \mid A D E)$, $P(D \mid A C E)$ and $P(E \mid A C D)$. As a result, a set of MCPDs with respect to the frequent itemset $A C D E$ is obtained, i.e., $P(A \mid C E), P(C \mid A E), P(D \mid E), P(D \mid C), P(D \mid A)$ and $P(E \mid A C)$, where $P(A \mid C E)$ is a MCPD of $P(A \mid C D E)$ with respect to $A$, and $P(C \mid A E)$ is a MCPD of $P(C \mid$ $A D E$ ) with respect to $C$, etc. $\square$

From the MCPDs corresponding the frequent itemset $X$, we can readily obtain a class of basic association rules, $C_{r}(X)$. A class of basic association rules derived from one frequent itemset may be completely included in another class of basic association rules derived from another frequent itemset. Hence, classes of basic association rules that are completely contained in other classes of basic association rules have no more information than the classes containing them. They are called redundant classes and are discarded.

Example 3.2.2. Given the transaction dataset in Table 2.1, minsup $=0.3$, and minconf $=0.6$, from Example 3.2.1, we obtain $C_{r}(A C D E)=\{C E \rightarrow A, A E \rightarrow C, E \rightarrow D$, $C \rightarrow D, A \rightarrow D, A C \rightarrow E\}$. Similarly, we also obtain another class of basic association rules corresponding to the frequent itemset $A D E, C_{r}(A D E)=\{A \rightarrow D, E \rightarrow D\}$. Since $C_{r}(A D E) \subset C_{r}(A C D E), C_{r}(A D E)$ is discarded.

\subsection{The GenBR Algorithm}

We propose the GenBR algorithm for generating $B R$. Its goal is different from that of the second step of the Apriori algorithm [1], which generates all association rules. Our approach consists of two main steps. Given a set of frequent itemsets and minconf, GenBR generates all classes of basic association rules. Secondly, the algorithm generates $B R$ by discarding all redundant classes.

The GenBR algorithm, presented in Figure 3.3, generates BR from a set of frequent itemsets $L$. For each frequent itemset $I$ in $L$, the GenBC algorithm is called to generate a class of basic association rules corresponding to $I$. All classes discovered by GenBC are collected into

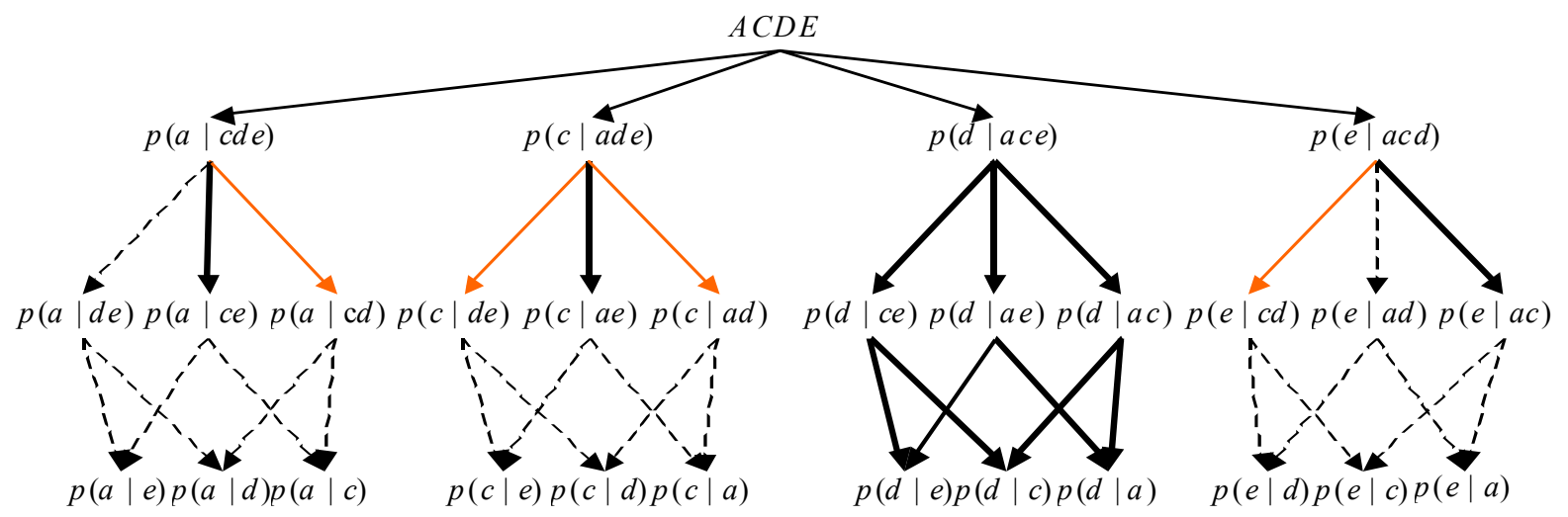

Figure 3.2. The Computation of MCPDs. 
Algorithm GenBR $(L)$

Purpose: generate $B R$ from a set $L$ of frequent itemsets

Input: $L$, a set of frequent itemsets, where $L^{k} \subseteq L$ is all itemsets in $L$ containing $k$ items.

Output: $B R$, a set of classes of basic association rules. begin

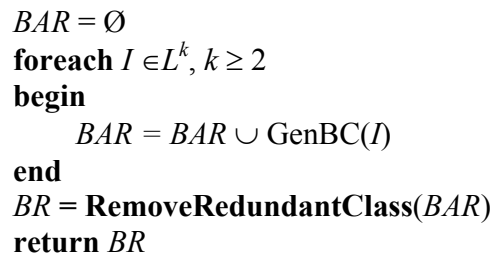

Figure 3.3. The GenBR Algorithm.

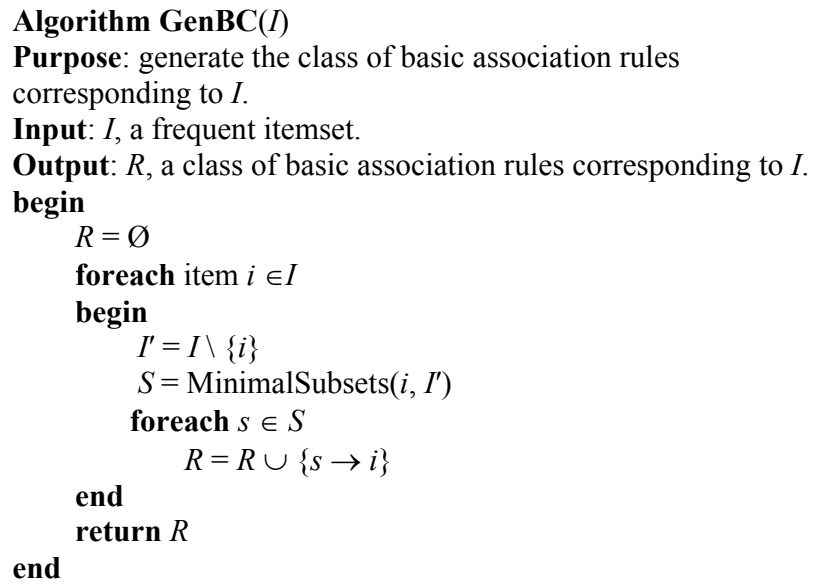

\section{Figure 3.4. The GenBC Algorithm.}

BAR. The RemoveRedundantClass algorithm removes redundant classes from BAR to give BR.

The GenBC algorithm, presented in Figure 3.4, generates the class of basic association rules corresponding to the frequent itemset $I$. The main loop of the GenBC algorithm is repeated for each item $i$ in the frequent itemset $I$. It calls the MinimalSubsets algorithm for computing the MCSs of $I^{\prime}$ with respect to $i$. From these MCSs, the algorithm forms a set of basic association rules corresponding to $I$.

The MinimalSubsets algorithm, presented in Figure 3.5 , computes the set of MCSs of $I^{\prime}$ with respect to $i$. First, the algorithm determines whether $p=p\left(i \mid I^{\prime}\right) \geq$ minconf. If so, the algorithm initializes the minimal conditional subset $S=\left\{I^{\prime}\right\}$. The MaximalSubsets function produces a set $S^{\prime}$ of all maximal subsets of $I^{\prime}$ as a set of candidate MCSs of $I^{\prime}$ with respect to $i$, e.g., $S^{\prime}=$ MaximalSubsets $(B D E)=\{B D, B E, D E\}$. Because this function is straightforward, we omit it. In the while loop, the algorithm examines the validity of candidate MCSs in $S^{\prime}$. For $\forall s \in S$, the algorithm computes the conditional probability $p(i \mid s)$, and then compares $p(i \mid s)$ with $p$. If $p(i \mid s)=p$, then $s$ is a valid candidate MCS of $I^{\prime}$ with respect to $i$, and it is stored in $S$. If smaller valid candidate
Algorithm MinimalSubsets $\left(i, I^{\prime}\right)$

Purpose: compute a set $S$ of minimal conditional subsets of $I^{\prime}$ with respect to $i$.

Input: $i$, an item such that $I^{\prime} \cup i$ is a frequent itemset.

$I^{\prime}$, an itemset.

Output: $S$, a set of minimal conditional subsets of

$I^{\prime}$ with respect to $i$.

begin

$S=\varnothing$

$p=\operatorname{supp}\left(I^{\prime} \cup\{i\}\right) / \operatorname{supp}\left(I^{\prime}\right)$

if ( $p \geq$ minconf) then

$S=\left\{I^{\prime}\right\}$

$S^{\prime}=\operatorname{MaximalSubsets}\left(I^{\prime}\right)$

$k=2$

while $\left(S^{\prime} \neq \varnothing\right)$

begin

foreach $s \in S^{\prime}$

begin

$p_{1}=\operatorname{supp}(s \cup\{i\}) / \operatorname{supp}(s)$

if $\left(p_{1}=p\right)$ then $S=S \cup\{s\}$

else $S^{\prime}=S^{\prime} \backslash\{s\}$

end

foreach $s \in S^{\prime}$

$\operatorname{DelSuperset}(s, S) / /$ remove all $s^{\prime} \in S$ such that $s^{\prime} \supset s$ from $S$

$S^{\prime}=\operatorname{IntersectionSet}\left(S^{\prime}, k\right)$

$k=k+1$

end

return $S$

end

Figure 3.5. The MinimalSubsets Algorithm.

MCSs of $I^{\prime}$ are found, then supersets of them are removed from $S$. The DelSuperset function (omitted) does this task. The IntersectionSet $\left(S^{\prime}, k\right)$ function (omitted) generates all smaller candidate MCSs of $I^{\prime}$, which are the intersections of itemsets in $S^{\prime}$ in terms of the depth $k$ of loop. For example, the intersection of the two itemsets $A E$ and $A C$ is equal to $A$, and it is regarded as a candidate MCS.

Example 3.3.1. Given the dataset in Table 2.1, minsup = 0.3 , and minconf $=0.6$, we describe the process of generating $B R$ using GenBR. In the while loop of GenBR, we assume $I=\{A C D E\}$ is selected from $L$. The GenBC algorithm is called to compute the class of basic association rules corresponding to $A C D E$.

The main loop of the GenBC algorithm is repeated for each item in a frequent itemset. Inside the main loop, the MinimalSubsets algorithm is first called to generate all MCSs of $C D E$ with respect to $A$. $I^{\prime}=C D E$ and $i=A$. Because the positive conditional probability $p(a \mid c d e) \geq$ minconf, the MinimalSubsets algorithm begins computing MCPDs of $P(A \mid C D E)$, i.e., a set $S$ of MCSs of $C D E$ with respect to $A$. Initially, $S=\left\{I^{\prime}\right\}$. MaximalSubsets produces a set $S^{\prime}$ of all maximal subsets of $C D E$ as candidate MCSs of 
$C D E$ with respect to $A, S^{\prime}=\{D E, C E, C D\}$. In the while loop, itemsets in $S^{\prime}$ are checked to see if they are candidate MCSs of $C D E$ with respect to $A$. When $C E$ is examined, $p(a \mid c e)=p(a \mid c d e)$, so we obtain $S=\{B D E, C E\}$. For $D E$ and $C D$, because $p(a \mid d e) \neq p(a \mid c d e)$ and $p(a \mid c d) \neq p(a \mid$ cde), $D E$ and $C D$ are removed from $S^{\prime}$, and $S^{\prime}=\{C E\}$. After DelSuperset, $S=\{C E\}$ and $S^{\prime}=\{C E\}$. Because $S^{\prime}$ has only one itemset, $S^{\prime}=$ IntersectionSet $\left(S^{\prime}, k\right)=\varnothing$. The while loop in the MinimalSubsets algorithm exits, and $S=$ $\{C E\}$ is returned to GenBC. In GenBC, the basic association rule $\{C E \rightarrow A\}$ is placed in $R$.

Similarly, from the computation of $P(C \mid A D E)$, we have a new basic association rule $A E \rightarrow C$, and $R=$ $\{C E \rightarrow A, A E \rightarrow C\}$. From the computation of $P(D \mid A C E)$, we have new basic association rules $E \rightarrow D, C \rightarrow D$ and $A \rightarrow D$, and they are added into $R$. From the computation of $P(E \mid A C D)$, a new basic association rule $A C \rightarrow E$ is found.

Finally, the algorithm generates a class of basic association rules corresponding to $A C D E, R=\{C E \rightarrow A$, $A E \rightarrow C, E \rightarrow D, C \rightarrow D, A \rightarrow E, A C \rightarrow E\}$. The algorithm returns $R$ to GenBR. At this point, $B R=\{\{D E \rightarrow A, A \rightarrow B$, $D \rightarrow B, E \rightarrow B, A \rightarrow E\}\}$.

GenBR continues until all frequent itemsets in $L$, with size of at least 2, have been processed. Consequently, all basic association rules and their classes are generated and presented, as shown in Table 3.1.

We define the following terminology over $B R$ :

(1) $C_{r}$ denotes a class of basic association rules. $C_{i}$ is a set of items, which appear in $C_{r}$.

(2) The class in which a basic association rule $X \rightarrow Y$ resides is denoted as $C_{r}(X \rightarrow Y)$. For example, $C_{r}(C \rightarrow D)$ might be referred to as one of $C_{r}(C D E), C_{r}(A C D), C_{r}(A C D E)$ and $C_{r}(C D)$, as shown in Table 3.1.

(3) $C_{i}(X \rightarrow Y)$ denotes a set of items, which appear in $C_{r}(X \rightarrow Y)$. e.g, $E \in C_{i}(C \rightarrow D)=\{C, D, E\}$.

All redundant classes in Table 3.1 are discovered and discarded by the RemoveRedundantClass algorithm. A

Table 3.1. A Set of Classes of Basic Association Rules.

\begin{tabular}{|c|l|l|c|}
\hline No. & \multicolumn{1}{|c|}{ Class } & \multicolumn{1}{c|}{ Basic association rule } & Redundant \\
\hline 1 & $C_{r}(A B)$ & $B \rightarrow A$ & Yes \\
\hline 2 & $C_{r}(B D)$ & $B \rightarrow D$ & Yes \\
\hline 3 & $C_{r}(D E)$ & $E \rightarrow D, D \rightarrow E$ & \\
\hline 4 & $C_{r}(A D)$ & $D \rightarrow A, A \rightarrow D$ & Yes \\
\hline 5 & $C_{r}(C E)$ & $E \rightarrow C, C \rightarrow E$ & Yes \\
\hline 6 & $C_{r}(A C)$ & $A \rightarrow C, C \rightarrow A$ & Yes \\
\hline 7 & $C_{r}(C D)$ & $D \rightarrow C, C \rightarrow D$ & \\
\hline 8 & $C_{r}(A B D)$ & $B \rightarrow D, B \rightarrow A, D \rightarrow A, A \rightarrow D$ & \\
\hline 9 & $C_{r}(A D E)$ & $E \rightarrow D, A \rightarrow D$ & Yes \\
\hline 10 & $C_{r}(A C E)$ & $C E \rightarrow A, A C \rightarrow E, A E \rightarrow C$ & Yes \\
\hline 11 & $C_{r}(C D E)$ & $E \rightarrow D, E \rightarrow C, C \rightarrow E, C \rightarrow D$ & \\
\hline 12 & $C_{r}(A C D)$ & $A \rightarrow D, A \rightarrow C, C \rightarrow D, C \rightarrow A$ & \\
\hline 13 & $C_{r}(A C D E)$ & $E \rightarrow D, C E \rightarrow A, A C \rightarrow E, A \rightarrow D$, & \\
& & $A E \rightarrow C, C \rightarrow D$ & \\
\hline
\end{tabular}

redundant class $C_{r}(I)$ contains no more information than the class $C_{r}\left(I^{\prime}\right)$ containing $C_{r}(I)$. For example, in Table 3.1, $C_{r}(A B) \subset C_{r}(A B D)$. If a $C_{r}\left(I_{1}\right)$ is redundant and $C_{r}\left(I_{1}\right)$ $\subset C_{r}\left(I_{2}\right)$, then $I_{1} \subset I_{2}$.

All frequent itemsets are arranged in a semi-lattice based on their inclusion relation in order to discover all redundant classes. For example, given the dataset shown in Table 2.1 and minsup $=0.3$, all frequent itemsets form the semi-lattice shown in Figure 3.6. To identify the redundant class $C_{r}(A B), \quad$ RemoveRedundantClass compares $C_{r}(A B)$ with $C_{r}(A B D)$ because $A B \subset A B D$. To check whether $C_{r}(D E)$ is redundant, the algorithm compares $C_{r}(D E)$ with $C_{r}(A D E)$ and $C_{r}(C D E)$. Because $C_{r}(D E) \not \subset C_{r}(A D E)$ and $C_{r}(D E) \not \subset C_{r}(C D E)$, the algorithm does not need to compare $C_{r}(D E)$ with $C_{r}(A C D E)$, and $C_{r}(D E)$ is non-redundant. That means that to check whether $C_{r}\left(I_{1}\right)$ is redundant, we only compare $C_{r}\left(I_{1}\right)$ with all $C_{r}\left(I_{2}\right)$, where $I_{1}$ and $I_{2}$ are frequent itemsets, $I_{1} \subset I_{2}$, and $\left|I_{1}\right|=\left|I_{2}\right|-1$, as justified by Theorem 3.3.1.

Theorem 3.3.1. Given three itemsets $I_{1}, I_{2}$, and $I_{3}$, and $I_{1}$ $\subset I_{2} \subset I_{3}$, if $C_{r}\left(I_{1}\right) \not \subset C_{r}\left(I_{2}\right)$, then $C_{r}\left(I_{1}\right) \not \subset C_{r}\left(I_{3}\right)$.

Proof: Suppose the condition is satisfied. Hence, $\exists r$ : $X \rightarrow A \in C_{r}\left(I_{1}\right)$, and a $\mathrm{MCP} p(a \mid x)$, but $r \notin C_{r}\left(I_{2}\right)$, i.e., $\exists Z$ $\subseteq I_{2} \backslash\{X, A\}, p(a \mid x) \neq p(a \mid x, z)$. Because $I_{2} \backslash\{X, A\} \subset I_{3}$ $\backslash\{X, A\}, \exists Z \subseteq I_{3} \backslash\{X, A\}, p(a \mid x) \neq p(a \mid x, z)$, i.e., $P(A \mid$ $X)$ is not a MCPD of $P\left(A \mid I_{3} \backslash A\right)$. Hence, $r \notin C_{r}\left(I_{3}\right)$.

In [22], we proposed an inference system for basic association rules. It is called the $C$-inference system, because it permits a rule's confidence to be inferred. We proved that the $C$-inference system holds on $B R$ and that all association rules can be derived from $B R$ by the application of inference rules in the $\mathrm{C}$-inference system. The C-inference system is summarized in Table 3.2, where $p$ and $q$ signify the confidences of association rules. Rules that cannot be derived from other rules by the Cinference system are called non-redundant association rules.

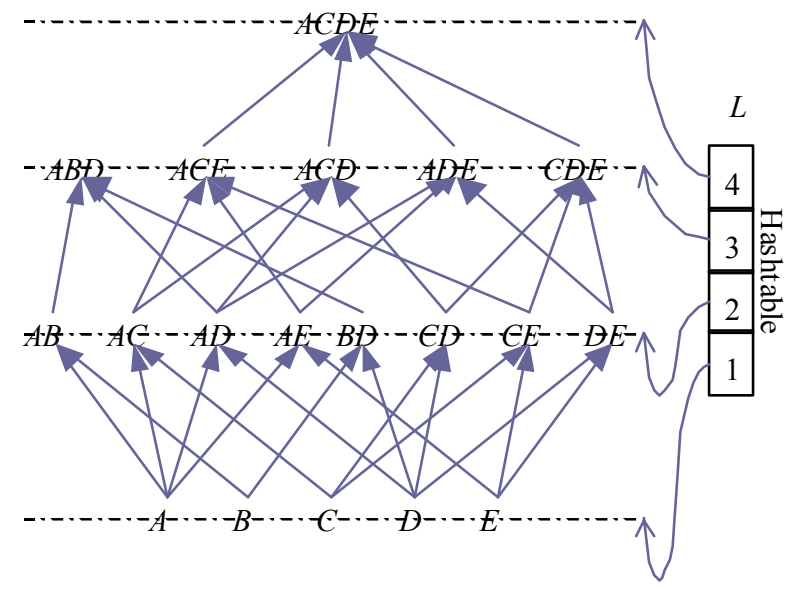

Figure 3.6. A Semi-Lattice for All Frequent Itemsets 
Table 3.2. The $C$-inference System on Basic Association Rules

\begin{tabular}{|c|c|c|}
\hline Inference Rule & Premise & Conclusion \\
\hline Augmentation & $\stackrel{p}{\rightarrow} Y \in C_{r}, \forall X^{\prime} \subseteq C_{i} \backslash\{X, Y\}$ & $X X^{\prime} \stackrel{p}{\rightarrow} Y$ \\
\hline Pseudo-Transitivity & $\stackrel{p}{\longrightarrow} \underset{X}{\rightarrow} Y \in C_{r}, X W \stackrel{q}{\rightarrow} Z \in C_{r}$ & $\stackrel{q}{\rightarrow} Z$ \\
\hline Contraction & $\stackrel{p}{\rightarrow} Y \in A R, X Y \stackrel{q}{\rightarrow} Z \in A R$ & $\stackrel{p q}{\rightarrow} Y Z$ \\
\hline Additivity & $\stackrel{p}{\rightarrow} Y \in C_{r}, W \stackrel{q}{\rightarrow} Z \in C_{r}$ & $\stackrel{p q}{X W} Y Z$ \\
\hline Right union & $\stackrel{p}{\rightarrow} Y \in C_{r}, X \stackrel{q}{\rightarrow} Z \in C_{r}$ & $\begin{array}{l}p q \\
X \rightarrow Y Z\end{array}$ \\
\hline Left union & $\stackrel{p}{\rightarrow} Z \in C_{r}, Y \stackrel{p}{\rightarrow} Z \in C_{r}$ & $\stackrel{p}{\longrightarrow} Z$ \\
\hline
\end{tabular}

\subsection{The Computational Complexity of GenBR}

Let us analyze the computational complexity of GenBR. From Figure 3.1, we observed that itemsets contained in the context of RCPDs may be used to construct a specific semi-lattice for computing MCPDs. All nodes in the semi-lattice are subitemsets of an itemset.

This construction corresponds to the problem of generating subsets in mathematics, or creating a hypercube (or a $k$-cube), or a $Q_{n}$ graph in graph theory [15]. We analyze the computational complexity of our algorithm based on the binomial theorem [13] as follows.

According to the binomial theorem, the number of subitemsets of a $k$-itemset (nodes of the semi-lattice), denoted $N S$, is given by:

$$
N S=C^{1}{ }_{k}+\ldots+C^{k}{ }_{k}=2^{k}-1
$$

By counting edges from the top of the semi lattice for a $k$-itemset, as shown in Figure 3.7, to its bottom, the number of edges, denoted $N E$, of the semi-lattice is given by:

$$
\begin{aligned}
& N E=C_{k}^{k-1}+2 C_{k}^{k-2}+3 C_{k}^{k-3}+\ldots+(k-1) C_{k}^{1} \\
& =C_{k}^{k-1}+2 C_{k}^{k-2}+3 C_{k}^{k-3}+\ldots+(k-1) C_{k}^{1}+k C_{k}^{0}- \\
& =\sum_{i=1}^{k} i C_{k}^{k-i}-k=\sum_{i=1}^{k} \frac{i k !}{i !(k-i) !}-k \\
& =k \sum_{i=1}^{k} \frac{(k-1) !}{(i-1) !(k-i) !}-k \\
& =k \sum_{i=1}^{k} C_{i-1}^{k-1}=k 2^{k-1}-k
\end{aligned}
$$$$
k C_{k}^{0}
$$

From Equation (3.1), we obtain the number of association rules NR for a $k$-itemset generated by the FastGenRules algorithm [2] as follows:

$$
N R=2^{k}-2
$$

According to Figure 3.1, to compute the MCSs of $A B C D$ with respect to $E$, in the worst case, the process forms a semi-lattice of $A B C D$. One edge in the semilattice corresponds to one comparison. For the whole itemset $A B C D E$, there are five semi-lattices of this kind. Hence, from Equation (3.2), the computational complexity $T C$ of GenBR for a $k$-itemset is determined by:

$$
\begin{aligned}
T C & =k(k-1)\left(2^{k-2}-1\right) \approx k^{2} 2^{k-2} \\
& =O\left(k^{2} 2^{k-2}\right)
\end{aligned}
$$

Given that $l$ is the length of the longest itemsets in the set of frequent itemsets, the ratio of the complexity of GenBR to that of FastGenRules is:

$$
\begin{aligned}
R & =O\left(l^{2} 2^{l-2}\right) / O\left(2^{l+1}\right)=O\left(l^{2} / 2^{3}\right) \\
& =O\left(l^{2}\right)
\end{aligned}
$$

Although the time complexity of GenBR exponentially increases with $l$, we show that GenBR performs very well over actual datasets in the following section.

\section{Comparison and Experimental Results}

The overall comparison between our approach and four previous approaches is shown in Table 4.1.

Table 4.1. The Overall Evaluation

\begin{tabular}{|l|c|c|c|c|c|}
\hline \multicolumn{1}{|c|}{ Algorithm } & $\begin{array}{c}\text { Fast- } \\
\text { Gen- } \\
\text { Rules }\end{array}$ & $\begin{array}{c}\text { Fast- } \\
\text { Gen- } \\
\text { RR }\end{array}$ & $\begin{array}{c}\text { Gen- } \\
\text { GBRI }\end{array}$ & $\begin{array}{c}\text { Cover- } \\
\text { Rules }\end{array}$ & GenBR \\
\hline Canonical form & & & & & $\sqrt{ }$ \\
\hline Non-redundant & & & & & $\sqrt{ }$ \\
\hline Infer a rule's support & & & & & $\sqrt{ }$ \\
\hline Infer a rule's confidence & & & $\sqrt{ }$ & & $\sqrt{ }$ \\
\hline Armstrong axioms-like & & & & & Best \\
\hline \#Rules & All & Fewer & Most & Fewest & Fast \\
\hline Elapsed time & Fastest & Slower & Slowest & Medium & Fant \\
\hline
\end{tabular}

First, consider the form of rules generated. Only GenBR generates non-redundant rules in canonical form. We believe that this type of rules is easy for users to understand. Rules generated by GenBR are nonredundant, i.e., these rules and their confidences cannot be derived from simpler rules and their confidences by using the inference rules defined in any of the other approaches. Secondly, consider whether an inference rule permits the support and confidence of rules to be inferred. None of the approaches can infer a rule's support, and only GenGBRI and GenBR can infer confidences. Thirdly, consider whether the inference system resembles Armstrong's axioms; only the C-inference system does so. Fourthly, consider the number of rules generated. CoverRules generates the fewest, but GenBR organizes the basic association rules into classes related to the frequent itemsets. GenBR also tends to generate the fewest rules when minconf is high. Finally, with respect to the elapsed time, FastGenRules is the fastest on all datasets. GenBR is more efficient than the other approaches, except for a few cases.

As described in the remainder of this section, experiments on several synthetic and real-life datasets were conducted to compare the performance of GenBR and previous algorithms with respect to the number of rules and the elapsed running time. 


\subsection{Experimental Design}

The experimental environment was a $\mathrm{PC}$ with a 2.53 $\mathrm{GHz}$ Intel CPU, 512MB of RAM, and Microsoft Windows XP. All algorithms were implemented in Microsoft Visual Java++.

The datasets used are listed in Table 4.2. Synthetic datasets, T10I4D100K and T20I6D100K, were generated by running GenData [17], which is a synthetic data generator from IBM. Several well-known benchmark real-world datasets were chosen from [29]. CustInfo-5 was obtained from the CustInfo database [4] by joining five tables. The number of attributes shown for the realworld datasets represents the number after multi-valued attributes were converted to several binary attributes.

Table 4.2. Synthetic and Real-world Datasets

\begin{tabular}{|l|r|r|r|r|}
\hline \multicolumn{1}{|c|}{ Dataset } & \# Attributes & \#Items & $\begin{array}{r}\text { Length of } \\
\text { Record or } \\
\text { Transactions }\end{array}$ & \# Transactions \\
\hline Chess & 37 & 75 & 37 & 3196 \\
\hline Connect & 43 & 129 & 43 & 67557 \\
\hline Mushroom & 23 & 128 & 23 & 8124 \\
\hline Molecular & 62 & 483 & 62 & 3190 \\
\hline CustInfo-5 & 14 & 9168 & 14 & 908241 \\
\hline T10I4D100K & & 1000 & 10 & 100000 \\
\hline T20I6D100K & & 1000 & 20 & 100000 \\
\hline
\end{tabular}

To compare the performance of GenBR and previous approaches with respect to the number of rules and the elapsed running time, we implemented the FastGenRules for generating association rules [2] as well as the Gen-GB algorithm for generating generic basis for exact rules, and the Gen-RI algorithm for generating informative basis for approximate association rules [5]. The Gen-GB and the Gen-RI algorithms were combined into the Gen-GBRI algorithm for our experiments. The FastGenRepresentatives (denoted FastGenRR) algorithm generates a set $R R$ of representative association rules [20]. CoverRules generates an informative cover of cover rules [11].

The GenBR algorithm consists of two steps to generate basic association rules. The first step generates all classes of basic association rules. The second step removes redundant classes. The GenBR algorithm is more similar to the FastGenRules algorithm than to the other algorithms. To compare the GenBR with FastGenRules with respect to the elapsed running time, we only consider the first step of GenBR, which generates the basic association rules, and we ignore the elapsed time required for the second step.

\subsection{Results}

We first compared the algorithms with regard to the number of rules generated. GenBR generates fewer rules than FastGenRules, and in some cases fewer rules than FastGenRR, Gen-GBRI, and CoverRules.
Table 4.3. Chess, minsup $=80 \%$

\begin{tabular}{|l|l|r|r|r|r|r|r|}
\hline \multicolumn{2}{|l|}{ Algorithm / minconf } & \multicolumn{1}{|c|}{10} & \multicolumn{1}{c|}{30} & \multicolumn{1}{c|}{60} & \multicolumn{1}{c|}{80} & \multicolumn{1}{c|}{90} & \multicolumn{1}{c|}{100} \\
\hline $\begin{array}{l}\text { Fast- } \\
\text { Gen- } \\
\text { Rules }\end{array}$ & \#Rules & 552564 & 552564 & 552564 & 552564 & 349298 & 4192 \\
\cline { 2 - 8 } & $\begin{array}{l}\text { Elapsed time } \\
\text { (ms) }\end{array}$ & 39125 & 38594 & 39031 & 39000 & $\mathbf{2 0 6 4 0}$ & $\mathbf{9 6 9}$ \\
\hline $\begin{array}{l}\text { Gen- } \\
\text { GBRI }\end{array}$ & \#Rules & 27791 & 27791 & 27791 & 27791 & 26852 & 2228 \\
\cline { 2 - 8 } & Elapsed time & 93020 & 93047 & 93021 & 93020 & 93057 & 92458 \\
\hline $\begin{array}{l}\text { Fast- } \\
\text { Gen- } \\
\text { RR }\end{array}$ & \#Rules & 1678 & 1678 & 1678 & 1678 & 4378 & 2228 \\
\cline { 2 - 8 } & Elapsed time & 59234 & 58750 & 58719 & 58703 & 57656 & 60188 \\
\hline $\begin{array}{l}\text { Cover- } \\
\text { Rules }\end{array}$ & \#Rules & $\mathbf{2 2 6}$ & $\mathbf{2 2 6}$ & $\mathbf{2 2 6}$ & $\mathbf{2 2 6}$ & $\mathbf{1 7 0 2}$ & 2228 \\
\cline { 2 - 8 } & Elapsed time & 36453 & 36734 & 36437 & 36453 & 526922 & 211609 \\
\hline \multirow{2}{*}{ GenBR } & \#Classes & 8112 & 8112 & 8112 & 8112 & 7710 & 145 \\
\cline { 2 - 8 } & \#Rules & 28416 & 28416 & 28416 & 28416 & 27054 & $\mathbf{6}$ \\
\cline { 2 - 8 } & Elapsed time & $\mathbf{2 5 3 9 1}$ & $\mathbf{2 5 1 4 1}$ & $\mathbf{2 5 2 0 2}$ & $\mathbf{2 5 5 9 3}$ & 25266 & 16468 \\
\hline
\end{tabular}

For Chess with minsup $=80 \%$, the number of rules generated by the algorithms and their elapsed times are shown in Table 4.3.

GenBR generates significantly fewer rules than FastGenRules for all settings of minconf. For example, when minconf $=10 \%$, FastGenRules generates 552564 rules, while GenBR generates 28416 rules.

Among all approaches, CoverRules generates the fewest rules when minconf is $90 \%$ or less. For example, in Table 4.3, with minconf $=10 \%$, CoverRules generates 226 rules while GenBR generates 28416 rules. However, when minconf is $100 \%$, GenBR generates the fewest rules. For example, in Table 4.3, GenBR generates only 6 rules, while the other approaches generate 2228 or more rules.

Table 4.4. Comparison wrt the Number of Rules.

\begin{tabular}{|l|r|r|r|r|r|r|}
\hline Dataset & Minsup & $\begin{array}{c}\text { Fast- } \\
\text { Gen- } \\
\text { Rules }\end{array}$ & $\begin{array}{c}\text { Fast- } \\
\text { Gen- } \\
\text { RR }\end{array}$ & $\begin{array}{c}\text { Gen- } \\
\text { GBRI }\end{array}$ & $\begin{array}{c}\text { Cover- } \\
\text { Rules }\end{array}$ & GenBR \\
\hline Chess & $80 \%$ & 4192 & 2228 & 2228 & 2228 & $\mathbf{6}$ \\
\hline Chess & $90 \%$ & 132 & 116 & 116 & 116 & $\mathbf{2}$ \\
\hline Connect-4 & $95 \%$ & 2270 & 684 & 684 & 684 & $\mathbf{1 0}$ \\
\hline Connect-4 & $97 \%$ & 245 & 161 & 161 & 161 & $\mathbf{4}$ \\
\hline Mushroom & $30 \%$ & 8450 & 557 & 557 & 426 & $\mathbf{7 6}$ \\
\hline Mushroom & $40 \%$ & 939 & 169 & 169 & 139 & $\mathbf{4 3}$ \\
\hline Molecular & $5 \%$ & 2085 & 1261 & 1261 & 1221 & $\mathbf{3 9 1}$ \\
\hline Molecular & $10 \%$ & 57 & 45 & 45 & 43 & $\mathbf{2 2}$ \\
\hline CustInfo-5 & $5 \%$ & 4913 & 656 & 2591 & $\mathbf{4 7 5}$ & 2591 \\
\hline CustInfo-5 & $10 \%$ & 1862 & 330 & 1078 & $\mathbf{2 3 8}$ & 1078 \\
\hline T10I4D100K & $0.05 \%$ & 28695 & 6075 & 6075 & 4805 & $\mathbf{2 6 4 1}$ \\
\hline T10I4D100K & $0.1 \%$ & 657 & 522 & 522 & 501 & $\mathbf{1 3 4}$ \\
\hline T20I6D100K & $0.2 \%$ & 67 & 45 & 45 & 36 & $\mathbf{2 6}$ \\
\hline T20I6D100K & $0.3 \%$ & 33 & 11 & 11 & $\mathbf{3}$ & 12 \\
\hline
\end{tabular}

For other datasets and different parameters, the experimental results concerning the number of rules generated are similar to those shown in Table 4.3. We summarize these experimental results in Table 4.4. Because users are generally concerned with association rules with high confidences, we only describe the number of rules with $100 \%$ confidence $($ minconf $=100 \%$ ) for all datasets besides CustInfo-5. For CustInfo-5, since there is no exact association rule when minsup $=5 \%$ or $10 \%$, we use minconf $=90 \%$. Under these conditions, GenBR 
generates fewer rules than the other algorithms, except for a few cases when CoverRules generates the fewest.

Although the computation complexity of GenBR is exponentially greater than that of FastGenRules (as mentioned in Section 3.4), in our experiments, we observed that the elapsed time for GenBR is significantly less than that for FastGenRules, except for cases where both algorithms have their fastest performance, which correspond to values of minconf approaching $100 \%$. For example, in Table 4.3, when minconf is $10 \%$, the elapsed time for GenBR is $25391 \mathrm{~ms}$ while that of FastGenRules is $39125 \mathrm{~ms}$. When minconf is $100 \%$, the elapsed time of GenBR is $16468 \mathrm{~ms}$ while the elapsed time of FastGenRules is $969 \mathrm{~ms}$. Across all tested settings of minconf from $10 \%$ to $100 \%$, GenBR has the lowest maximum elapsed time (25593 $\mathrm{ms})$.

The number of exact association rules greatly affects the elapsed time of GenBR. If there are more exact association rules, there may be more functional dependencies, and consequently, GenBR spends more time.

We summarize the experimental results related to elapsed time by recording the ratios of the elapsed time of GenBR to previous algorithms in Table 4.5. We set minconf $=100 \%$. The bold entries identify cases where the corresponding algorithm is faster than GenBR. The results show that GenBR is faster than all algorithms except FastGenRules on most presented datasets.

Table 4.5. Evaluation wrt Elapsed Time

\begin{tabular}{|l|r|r|r|r|r|}
\hline Dataset & Minsup & $\begin{array}{c}\text { FastGen- } \\
\text { Rules }\end{array}$ & $\begin{array}{r}\text { FastGen- } \\
\text { RR }\end{array}$ & $\begin{array}{c}\text { Gen- } \\
\text { GBRI }\end{array}$ & $\begin{array}{c}\text { Cover- } \\
\text { Rules }\end{array}$ \\
\hline Chess & $80 \%$ & $\mathbf{1 6 . 9 9}$ & 0.27 & 0.18 & 0.08 \\
\hline Chess & $90 \%$ & $\mathbf{1 . 9 8}$ & 0.19 & 0.12 & 0.09 \\
\hline Connect-4 & $95 \%$ & $\mathbf{1 1 . 6 9}$ & 0.65 & $\mathbf{1 . 1 2}$ & 0.18 \\
\hline Connect-4 & $97 \%$ & $\mathbf{2 . 3 2}$ & 0.35 & 0.35 & 0.15 \\
\hline Mushroom & $30 \%$ & $\mathbf{3 0 . 4 7}$ & $\mathbf{2 . 5 1}$ & $\mathbf{1 6 . 7 2}$ & 0.66 \\
\hline Mushroom & $40 \%$ & $\mathbf{1 0 . 9 6}$ & $\mathbf{1 . 7 3}$ & $\mathbf{6 . 6}$ & 0.7 \\
\hline Molecular & $5 \%$ & $\mathbf{1 . 7 4}$ & 0.007 & 0.002 & 0.004 \\
\hline Molecular & $10 \%$ & $\mathbf{2 . 0 2}$ & 0.08 & 0.02 & 0.31 \\
\hline CustInfo-5 & $5 \%$ & $\mathbf{2 . 7 3}$ & 0.37 & 0.1 & 0.11 \\
\hline CustInfo-5 & $10 \%$ & $\mathbf{2 . 5 9}$ & 0.58 & 0.19 & 0.18 \\
\hline T10I4D100K & $0.05 \%$ & $\mathbf{1 5 . 8 6}$ & 0.05 & 0.01 & 0.08 \\
\hline T10I4D100K & $0.1 \%$ & $\mathbf{3 . 3 2}$ & 0.06 & 0.01 & 0.08 \\
\hline T20I6D100K & $0.2 \%$ & $\mathbf{2 . 1 2}$ & 0.03 & 0.002 & 0.07 \\
\hline T20I6D100K & $0.3 \%$ & $\mathbf{2}$ & 0.31 & 0.006 & $\mathbf{1 . 3}$ \\
\hline
\end{tabular}

\section{Conclusions and Future Work}

\subsection{Conclusions}

In this paper, we proposed a new type of association rules, called basic association rules.

First, by referring the relational database theory on functional dependencies, we developed the new concepts of a restricted conditional probability distribution (RCPD), a minimal conditional probability distribution (MCPD), a minimal conditional subset (MCS), and a basic association rule. We established the $C$-inference system on basic association rules, which is similar to Armstrong's axioms on functional dependencies.

Secondly, we proposed the GenBR algorithm for generating a set of classes of basic association rules from a set of frequent itemsets. GenBR efficiently generates basic association rules as compared with the previous approaches for generating small sets of association rules. GenBR also generates fewer rules than previous approaches when minconf is high.

Thirdly, arguably, users will find basic association rules to be more manageable and understandable than previously proposed reduced sets of association rules. The rules are concise, the number of rules is small, redundancy among rules in a class of basic association rules has been eliminated, and inference involving confidence values is possible on the rules. This point is argued at greater length in [22].

Fourthly, we showed that the search space of our algorithm to compute basic association rules is a hypercube ( $n$-cube) or $Q_{n}$ graph. This insight aided in our theoretical analysis of the algorithm.

\subsection{Future Work}

An open problem is to find a more efficient algorithm for discovering basic association rules from frequent itemsets. Finding a heuristic method to discover basic association rules without generating frequent itemsets will also be challenging.

We proposed the idea of a restricted conditional probability distribution as a foundation for mining association rules, and we distinguished it from the traditional conditional probability distribution. It can be regarded as a more general concept than the contextspecific conditional probability distribution described in [6]. Further work on restricted conditional probability distributions may be promising.

We established the $C$-inference system on association rules, which may be regarded as an extension of Armstrong's axioms on functional dependencies. This kind of inference system works on exact and approximate rules simultaneously. Further exploration of the properties of the $C$-inference system is needed.

\section{References}

[1] R. Agrawal, T. Imielinski, and A. Swami. Mining association rules between sets of items in large databases. In Proc. SIGMOD'93, pages 207-216, Washington. DC, May 1993.

[2] R. Agrawal and R. Srikant. Fast algorithm for mining association rules. In Proc. VLDB'94, Santiago, Chile, 1994.

[3] E. Baralis and G. Psaila. Designing templates for mining association rules. Journal of Intelligent Information Systems, 9(1):7-32, July 1997.

[4] B. Barber and H. J. Hamilton. Extracting share frequent itemsets with infrequent subsets. Data 
Mining and Knowledge Discovery, 7(2):153-185, April 2003.

[5] Y. Bastide, N. Pasquier, R. Taouil, G. Stumme, and L. Lakhal. Mining minimal non-redundant association rules using frequent closed itemsets. In Proc. of the First International Conference on Computational Logic, pages 972-986, 2000.

[6] C. Boutilier, N. Friedman, M. Goldszmidt, and D. Koller. Context-specific independence in Bayesian networks. In Proc. $12^{\text {th }}$ Conf. on Uncertainty in Artificial Intelligence (UAI-96), pages 115-123, Portland, Oregon, 1996.

[7] S. Brin, R. Motwani, and C. Silverstein. Beyond market baskets: Generalizing association rules to correlation. In Proc. SIGMOD'97, pages 265-276, May 1997.

[8] S. Brin, R. Motwani, J. D. Ullman and S. Tsur. Dynamic itemset counting and implication rules for market basket data. In Proc. SIGMOD'97, pages 255-264, Montreal, Canada, June 1997.

[9] C. L. Carter, H. J. Hamilton, and N. Cercone. Share based measures for itemsets. In Proc. PKDD'97, pages 14-24, Trondheim, Norway, June 1997.

[10] E. Castillo, J. M. Gutierrez, and A. S. Hadi, Expert Systems and Probabilistic Network Models, Springer, 1997.

[11] L. Cristofor and D. Simovici. Generating an informative cover for association rules. In Proc. of the IEEE International Conference on Data Mining, 2002.

[12] B. A. Davey and H. A. Priestley. Introduction to Lattices and Order, Fourth edition. Cambridge University Press, 1994.

[13] E. G. Goodaire and M. M. Parmenter. Discrete Mathematics with Graph Theory, Second Edition. Prentice Hall, Upper Saddle River, NJ, 2002.

[14] J. Han, J. Pei, and Y. Yin. Mining frequent patterns without candidate generation. In Proc. SIGMOD'00, pages 1-12, Dallas, TX, May 2000.

[15] T. W. Haynes, S. T. Hedetniemi, and P. J. Slater. Fundamentals of Domination in Graphs. Marcel Dekker, 1998.

[16] R. J. Hilderman and H. J. Hamilton. Knowledge Discovery and Interest Measures, Kluwer Academic, Boston, 2002.

[17] IBM. GenData, www.almaden.ibm.com/cs/quest/syndata.html

[18] M. Kamber and R. Shinghal. Evaluating the interestingness of characteristic rules. In Proc. KDD'96, pages 263-266, Portland, Oregon, 1996.

[19] M. Klemettinen, H. Mannila, P. Ronkainen, H. Toivnen, and A. I. Verkamo. Finding interesting rules from large sets of discovered association rules. In Proc. of the $3^{\text {rd }}$ CIKM Conference, pages 401-407, November 1994.
[20] M. Kryszkiewicz. Representative association rules and minimum condition maximum consequence association rules. In Proc. PKDD'98. pages 361369, Nantes, France, 1998.

[21] W. Lee, S. J. Stolfo, and K. W. Mokl. A data mining framework for building intrusion detection models. In Proc. IEEE Symposium on Security and Privacy, 1999.

[22] G. Li. Basic Association Rules, M.Sc. Thesis, Department of Computer Science, University of Regina, 2004.

[23] W. Lin, S. A. Alvarez, and C. Ruiz. Collaborative recommendation via adaptive association rules mining. In Proc. WEBKDD'2000, San Francisco, CA, Aug. 2000.

[24] N. Pasquier, Y. Bastide, R. Taouil, and L. Lakhal. Closed set based discovery of small covers for association rules. In Proc. of the $15^{\text {th }}$ Conference on Advanced Databases, pages 361-381, 1999.

[25] C. M. Ricardo. Database Systems: Principles, Design, \& Implementation, Macmillan, 1990.

[26] K. Satou, G. Shibayama, T. Ono, Y. Yamamura, E. Furuichi, S. Kuhara, and T. Takagi. Finding association rules on heterogeneous genome data. In Proc. of the Pacific Symposium on Biocomputing'97 (PSB'97), pages 397-408, Hawaii, Jan. 1997.

[27] R. Srikant, Q. Vu, and R. Agrawal. Mining association rules with item constraints. In Proc. $K D D^{\prime} 97$, pages 67-73.

[28] H. Toivonen, M. Klemettinen, P. Ronkainen, K. Hatonen, and H. Mannila. Pruning and grouping discovered association rules. In Proc. ECML-95 Workshop on Statistics, Machine Learning, and Knowledge Discovery in Database, pages 47-52, April 1995.

[29] UCI Machine Learning Repository. ftp://ftp.ics.uci.edu/pub/machine-learningdatabases/

[30] M. Zaki, Generating non-redundant association rules. In Proc. KDD'2000, pages 34-43. August 2000. 